\title{
The Value of Serum Midkine Level in Diagnosis of Hepatocellular Carcinoma
}

\author{
Karim Y. A. Shaheen, ${ }^{1}$ Abeer I. Abdel-Mageed, ${ }^{1}$ Eslam Safwat, ${ }^{2}$ and Ashraf M. AlBreedy ${ }^{3}$ \\ ${ }^{1}$ Department of Clinical and Chemical Pathology, Faculty of Medicine, Ain Shams University, Cairo 11566, Egypt \\ ${ }^{2}$ Internal Medicine Department, Faculty of Medicine, Ain Shams University, Cairo 11566, Egypt \\ ${ }^{3}$ Tropical Medicine Department, Faculty of Medicine, Ain Shams University, Cairo 11566, Egypt \\ Correspondence should be addressed to Ashraf M. AlBreedy; drashrafbreedy@yahoo.com
}

Received 29 September 2014; Accepted 19 January 2015

Academic Editor: Shigeru Marubashi

Copyright (C) 2015 Karim Y. A. Shaheen et al. This is an open access article distributed under the Creative Commons Attribution License, which permits unrestricted use, distribution, and reproduction in any medium, provided the original work is properly cited.

\begin{abstract}
Background and Aim. Identification of sensitive biomarkers to improve early diagnosis of HCC is needed. We aimed to evaluate serum midkine (MDK) as a biomarker for HCC diagnosis. Patients and Methods. 40 HCCs, 30 liver cirrhosis patients, and 30 healthy subjects were enrolled. Serum MDK using ELISA was measured in all included subjects. Results. Serum MDK was significantly elevated in HCC group compared to cirrhotic and healthy control groups ( 0.625 versus 0.15 and $0.125 \mathrm{ng} / \mathrm{mL})$, respectively. No significant association was found between MDK and either BCLC stage, tumor diameter, tumor number, or AFP level. Receiver operating characteristic curve showed that best cutoff for MDK and AFP was 0.387 and $88.5 \mathrm{ng} / \mathrm{mL}$, respectively. Area under the curve of MDK was significantly larger than that of AFP (0.941 versus 0.671 ). The sensitivity of MDK at $0.387 \mathrm{ng} / \mathrm{mL}$ for HCC diagnosis was significantly higher than that of AFP at cutoffs $20,88.5$, and $200 \mathrm{ng} / \mathrm{mL}$ (92.5 versus 62.5, 40, and 25\%), respectively. Sensitivity of MDK reached $93.3 \%$ in patients with AFP $<20 \mathrm{ng} / \mathrm{mL}$. Moreover, MDK at $0.387 \mathrm{ng} / \mathrm{mL}$ had significant better sensitivity than AFP at $20 \mathrm{ng} / \mathrm{mL}$ in distinguishing HCC from BCLC 0/A (90 versus 40\%). Conclusion. Serum MDK might be a potential diagnostic marker for HCC particularity in its early stages.
\end{abstract}

\section{Introduction}

Hepatocellular carcinoma (HCC) is the sixth most common cancer worldwide and the third most frequent cause of cancer-related death [1]. In Egypt, HCC represents $75 \%$ of malignant liver tumors. Liver cancer is the 5th most common cancer in both genders, the 6th in female representing 3.4\% of cancers and 2nd in order in males after cancer urinary bladder representing $11.5 \%$ of all cancers. In 2010, liver cancer came in the $3 \mathrm{rd}$ order in both sexes (8.1\%), 1st in males (12.1\%) and 5 th in females (4\%) [2].

HCC is a condition which lends itself to surveillance as at-risk individuals can readily be identified because of the presence of underlying viral hepatitis or other liver diseases. The aim of surveillance is to obtain a reduction in diseaserelated mortality. This is usually achieved through an early diagnosis (stage migration) that, in turn, enhances the applicability and cost effectiveness of curative therapies [3].
The imaging test most widely used for surveillance is ultrasonography (US). Alpha-fetoprotein (AFP) is the most widely tested biomarker in HCC. However, AFP has a suboptimal performance as a serological test for surveillance for 2 reasons; firstly, fluctuating levels of AFP in patients with cirrhosis might reflect flares of $\mathrm{HBV}$ or $\mathrm{HCV}$ infection, exacerbation of underlying liver disease or HCC development [4]. Secondly, only a small proportion of tumors at an early stage (10-20\%) present with abnormal AFP serum levels [5, 6]. In addition, other studies showed that des- $\gamma$-carboxyprothrombin [7] and AFP-L3 [8] were not superior to AFP for the diagnosis of early HCC. This highlights the need for new more reliable noninvasive recent biomarkers with better sensitivity and specificity for early diagnosis of HCC.

Midkine (MDK), also known as neurite growth-promoting factor 2 (NEGF2), is a basic heparin-binding growth factor of low molecular weight. In humans, it is encoded by the MDK gene on chromosome 11 [9]. It is a developmentally 
important retinoic acid-responsive gene product strongly induced during mid-gestation, hence the name midkine. Expression of the MDK gene in human adult tissues is extremely low and restricted. Mounting evidence has indicated that MDK plays a significant role in carcinogenesisrelated activities, such as proliferation, migration, antiapoptosis, mitogenesis, transformation, and angiogenesis, in many types of solid tumors, including hepatocellular carcinomas $[10,11]$. However, the diagnostic value of serum MDK for hepatocellular carcinomas, particularly for those at the early stage, has not yet been investigated in Egypt.

In this study we aimed to investigate the diagnostic utility of midkine in patients with newly diagnosed hepatocellular carcinoma (HCC).

\section{Subjects and Methods}

The study protocol conformed to medical research ethical guidelines. After approval of the Research and Ethics Committee of Ain Shams University, Cairo, Egypt, in accordance with local research governance requirements, the study was carried out at HCC Clinic, Clinical Pathology, and Tropical Medicine Departments, Ain Shams University Hospitals during the interval between September 2013 and April 2014. An informed consent was taken from each participant before enrollment in the study.

This study was conducted on 100 subjects who were divided into 3 groups; group 1 included 40 patients with newly diagnosed HCC, group 2 included 30 patients with liver cirrhosis (LC), and group 3 included age- and sex-matched apparently healthy subjects serving as a control group.

Liver cirrhosis was documented by clinical assessment, laboratory findings, and evidence of liver cirrhosis upon abdominal ultrasound. The diagnosis of HCC was confirmed according to American Association for the Study of Liver Diseases (AASLD) guidelines in 2011 [12]. Patients with previous HCC treatment and liver tumors other than HCC and those with Barcelona Clinic Liver Cancer (BCLC) stage D were excluded from the study.

The enrolled patients were subjected to full medical history taking, thorough clinical examination, and laboratory investigations including complete blood picture, complete liver and kidney profile, viral or autoimmune liver markers, serum alpha-fetoprotein by chemiluminescent immunometric technique, serum midkine by enzyme-linked immunosorbent assay (ELISA), and abdominal ultrasound. Only those in HCC group underwent further imaging in the form of abdominal triphasic spiral CT or magnetic resonance imaging as a part of HCC diagnosis. Child-Pugh classification was used to assess the severity of liver disease in patients with HCC and those with LC.

Serum MDK was measured in all enrolled subjects using ELISA kit supplied by Glory Science (Glory Science Co., 2400 Veterans Boulevard, Suite 16-101, Del Rio, TX 78840, USA). The assay is based on a double-antibody sandwich ELISA technique for the quantitative assay of human MDK in samples. In this technique, MDK binds to monoclonal antibody enzyme well which is precoated with human MK monoclonal antibody, making a solid phase antibody. Then MDK antibody is added and combines with Streptavidin-Horseradish Peroxidase (HRP) to form an immune complex. Following incubation, MDK is removed during a wash step and then substrates A and B are added to the wells and the color of the liquid changes into blue. The colored product is formed in proportion to the amount of MDK present in the sample. The reaction is terminated by addition of sulphuric acid. The concentration of MDK in the samples is then determined by comparing the (optical density) OD of the samples to the standard curve and values were reported as $\mathrm{ng} / \mathrm{mL}$.

Statistical analyses were conducted using SPSS 17.0 and MedCalc software. The significance level is 0.05 . Data were expressed as mean \pm SD for quantitative parametric measures in addition to Median Percentiles for quantitative nonparametric measures and both number and percentage for categorized data. The quantitative variables were analyzed by Mann-Whitney $U$ test $(Z)$. Kruskal Wallis test is applied for statistical comparison between more than two sets of data if one or both of them have a skewed distribution. Pearson correlation test $(r)$ was used to investigate the correlation between 2 quantitative variables. Chi square $\left(\chi^{2}\right)$ and Fisher's exact test $(F)$ were used to examine the relationship between Categorical variables. The ROC was constructed to obtain the most sensitive and specific cutoff value for serum MDK in diagnosing HCC. Logistic Regression Model was used to combine information of MDK and AFP for diagnosis of HCC.

\section{Results}

In this study, a total of 100 persons were recruited and enrolled including 40 newly diagnosed HCC patients with median age of 52 years old ( 30 were males (75\%) and 10 were females $(25 \%))$, 30 patients with liver cirrhosis with median age of 48 years old (18 males (60\%) and 12 females (40\%)), and 30 healthy individuals with median age of 45 years old (17 males (56.7\%) and 13 females (43.3\%)). Hepatitis C virus (HCV) was the underlying etiology of liver cirrhosis in 38 (95\%) and 29 (96.7\%) patients in HCC group and liver cirrhosis group, respectively, while only 2 (5\%) and 1 patients $(3.3 \%)$ in HCC group and liver cirrhosis group had chronic hepatitis $B$ virus infection, respectively. The HCC group included 20 patients with BCLC stages 0 and A (very early and early HCC), 10 patients with BCLC stage B (intermediate HCC) and 10 patients with BCLC stage C (advanced HCC). Child A class was found in 34 patients (24 in HCC group and 10 in liver cirrhosis group), Child B in 26 patients (16 in HCC group and 10 in liver cirrhosis group), and Child $\mathrm{C}$ in 10 patients with liver cirrhosis group.

The median values of the MDK levels in the HCC group were much higher when compared to the LC group $(0.625$ versus $0.15 \mathrm{ng} / \mathrm{mL} ; P<0.001)$ and to the healthy control group $(0.625$ versus $0.125 \mathrm{ng} / \mathrm{mL} ; P<0.001)$. Meanwhile, however, the median values of MDK levels in the LC group were higher than that in the control group, yet not reaching significance $(0.15$ versus $0.125 \mathrm{ng} / \mathrm{mL} ; P>0.05$ ) (Table 1 ). Regarding AFP, its median value was higher among HCC than LC group $(39.3 \mathrm{ng} / \mathrm{mL}$ versus $17.5 \mathrm{ng} / \mathrm{mL} ; P<0.05)$ 
TABLE 1: Comparison between each of the two studied groups regarding serums AFP and MDK.

\begin{tabular}{|c|c|c|c|c|c|c|c|c|}
\hline \multirow{2}{*}{ Parameters } & \multicolumn{2}{|c|}{ MDK (ng/mL) } & \multicolumn{2}{|c|}{ Significance } & \multicolumn{2}{|c|}{$\operatorname{AFP}(\mathrm{ng} / \mathrm{mL})$} & \multicolumn{2}{|c|}{ Significance } \\
\hline & Median & 1st and 3rd quartile & $Z$ & $P$ & Median & 1st and 3rd quartile & $Z$ & $P$ \\
\hline $\begin{array}{l}\text { HCC }(n=40) \text { versus } \\
\text { LC }(n=30)\end{array}$ & $\begin{array}{r}0.625 \\
0.15\end{array}$ & $\begin{array}{r}0.45-0.96 \\
0.12-0.23\end{array}$ & 6.278 & $\begin{array}{c}0 \\
\text { (HS) }\end{array}$ & $\begin{array}{r}39.3 \\
17.5\end{array}$ & $\begin{array}{c}13.5-225.5 \\
10-36\end{array}$ & 2.223 & $\begin{array}{l}0.026 \\
(S)\end{array}$ \\
\hline $\begin{array}{l}\text { HCC }(n=40) \text { versus } \\
\text { healthy }(30)\end{array}$ & $\begin{array}{r}0.625 \\
0.125\end{array}$ & $\begin{array}{c}0.45-0.96 \\
0.1-0.15\end{array}$ & 7.085 & $\begin{array}{c}0 \\
(\mathrm{HS})\end{array}$ & $\begin{array}{c}39.3 \\
1\end{array}$ & $\begin{array}{c}13.5-225.5 \\
0.5-2\end{array}$ & 6.914 & $\begin{array}{c}0 \\
(\mathrm{HS})\end{array}$ \\
\hline $\begin{array}{l}\text { LC }(n=30) \text { versus } \\
\text { healthy }(30)\end{array}$ & $\begin{array}{l}0.15 \\
0.125\end{array}$ & $\begin{array}{c}0.12-0.23 \\
0.1-0.15\end{array}$ & 1.559 & $\begin{array}{c}0.118 \\
(\mathrm{NS})\end{array}$ & $\begin{array}{c}17.5 \\
1\end{array}$ & $\begin{array}{r}10-36 \\
0.5-2\end{array}$ & 6.290 & $\begin{array}{c}0 \\
(\mathrm{HS})\end{array}$ \\
\hline
\end{tabular}

MDK: midkine, AFP: alpha fetoprotein, HCC: hepatocellular carcinoma, and LC: liver cirrhosis.

TABLE 2: The diagnostic performance of the best cutoff values of AFP and MDK for discriminating HCC group from LC group.

\begin{tabular}{ccccccrrr}
\hline & Cutoff value & AUC $(95 \% \mathrm{CI})$ & Sensitivity (\%) & Specificity (\%) & PPV (\%) & NPV (\%) & Accuracy (\%) & $P$ \\
\hline MDK & 0.387 & $\begin{array}{c}0.941 \\
(0.890-0.992)\end{array}$ & 92.5 & 83.3 & 88 & 89.2 & 88.5 & 0.001 \\
\hline AFP & 88.5 & $\begin{array}{c}0.671 \\
(0.546-0.796)\end{array}$ & 40 & 96.7 & 94.1 & 54.7 & 64.2 & 0.015 \\
\hline
\end{tabular}

MDK: midkine; AFP: alpha-fetoprotein; AUC; area under the curve; CI: confidence interval; PPV: positive predictive value; NPV: negative predictive value.

and healthy group $(39.3 \mathrm{ng} / \mathrm{mL}$ versus $1 \mathrm{ng} / \mathrm{mL} ; P<0.001)$ (Table 1).

Patients with BCLC stage B/C had significant higher median AFP levels $(300 \mathrm{ng} / \mathrm{mL})$ when compared to those with stage $0 / \mathrm{A}(17 \mathrm{ng} / \mathrm{mL})(P=0.001)$, while no significant association was found between serum MDK and BCLC stage $(0.526 \mathrm{ng} / \mathrm{mL}$ versus $0.75 \mathrm{ng} / \mathrm{mL} ; P=0.219)$. In the HCC group, there was no significant correlation between MDK with tumor diameter and number of tumor nodules $(r=$ $0.125 ; P=0.442$ and $r=0.129 ; P=0.427$, resp.). Also serum levels of MDK did not show any significant correlation with serum levels of AFP in all studied patients $(r=0.107 ; P=$ 0.291), while serum levels of AFP showed significant positive correlation only with tumor size but not with tumor number in the 40 HCC patients $(r=0.36 ; P=0.022$ and $r=0.088$; $P=0.588$, resp.). In addition, on comparing the median values of serum MDK in child classes $A, B$, and $C$ in CLD group patients using Kruskal Wallistest, there was no significant association found between serum MDK and child class $(K=5.936 ; P=0.061)$.

ROC curve was performed for the best cutoff point to differentiate between HCC group and LC group using MDK and AFP. Area under the curve (AUC) for serum MDK was 0.941 (95\% CI: 0.890-0.992) which was much higher when compared to that for AFP 0.671 (95\% CI: 0.546-0.796) with significant statistical difference $(P<0.001)$ (Figure 1). According to the curve, the best cutoff value for MDK differentiating HCC from LC cases was $0.387 \mathrm{ng} / \mathrm{mL}$, above which the sensitivity to discriminate HCC $=92.5 \%$ and below which the specificity to discriminate $\mathrm{LC}=83.3 \%$ with $88.5 \%$ accuracy (true results for both), while AFP could be used to differentiate HCC from LC cases at a cutoff level of $88.5 \mathrm{ng} / \mathrm{mL}$, with $40 \%$ sensitivity, $96.7 \%$ specificity, and $64.2 \%$ accuracy (Table 2).

To discriminate patients with HCC and those with LC, the sensitivity of MDK at cutoff value $0.387 \mathrm{ng} / \mathrm{mL}$ was found to be much significantly higher when compared to that of AFP

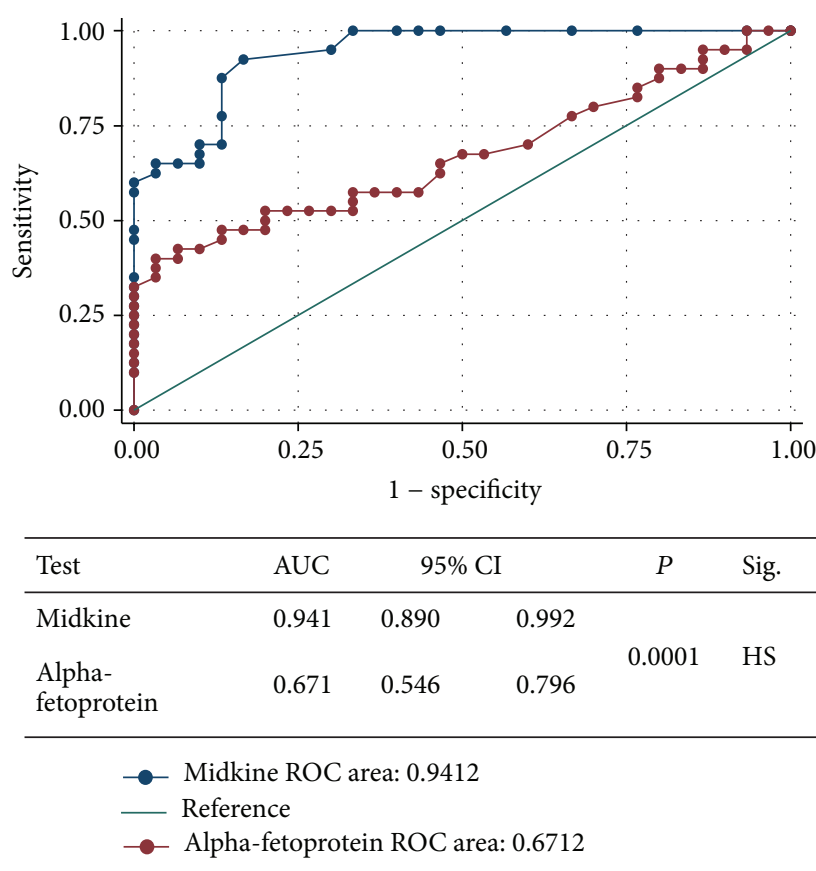

FIGURE 1: ROC curve showing comparison of the diagnostic performance of serum MDK and AFP for discriminating HCC group from chronic liver disease group.

at cutoff values $88.5,20$, and $200 \mathrm{ng} / \mathrm{mL}$ (92.5\% versus $40 \%$, $62.5 \%$, and $25 \%$, resp.) $P=0.001$, while the specificity of MDK cutoff value $0.387 \mathrm{ng} / \mathrm{mL}$ was only significantly higher than that of AFP at cutoff value $20 \mathrm{ng} / \mathrm{mL}$ ( $83.3 \%$ versus 53.3; $P=0.012$ ) (Table 3$)$.

To discriminate patients with early HCC (BCLC 0/A) from those with liver cirrhosis, the sensitivity of MDK at cutoff value $0.387 \mathrm{ng} / \mathrm{mL}$ was found to be much significantly higher when compared to that of AFP at cutoff values 20, 
TABLE 3: Comparison between MDK at cutoff value $0.387 \mathrm{ng} / \mathrm{mL}$ and AFP at cutoff values $88.5,20$, and $200 \mathrm{ng} / \mathrm{mL}$ regarding sensitivity and specificity in diagnosis of HCC.

\begin{tabular}{lccccc}
\hline & \multirow{2}{*}{ Cutoff } & \multicolumn{2}{c}{ Sensitivity } & \multicolumn{2}{c}{ Specificity } \\
& & $\%$ & $P$ & $\%$ & $P$ \\
\hline Midkine versus & 0.387 & 92.5 & $0.001^{*}$ & 83.3 & $0.195^{* *}$ \\
AFP & 88.5 & 40 & HS & $\mathbf{9 6 . 7}$ & NS \\
\hline Midkine versus & 0.387 & 92.5 & $0.001^{*}$ & 83.3 & $0.012^{*}$ \\
AFP & 20 & $\mathbf{6 2 . 5}$ & HS & $\mathbf{5 3 . 3 \%}$ & S \\
\hline Midkine versus & 0.387 & 92.5 & $0.001^{*}$ & 83.3 & $0.052^{* *}$ \\
AFP & 200 & $\mathbf{2 5 . 0 \%}$ & HS & 100 & NS \\
\hline
\end{tabular}

MDK: midkine; AFP: alpha-fetoprotein.

${ }^{*}$ Chi-square test.

${ }^{* *}$ Fisher's exact test.

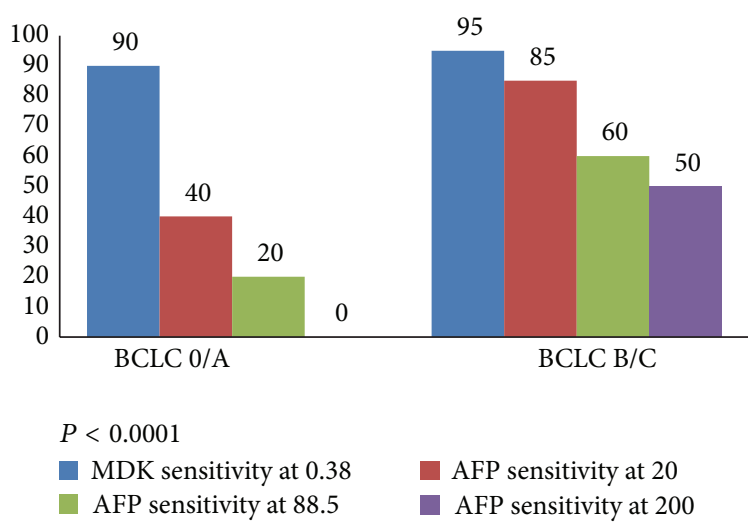

FIgURE 2: Sensitivity of MDK and AFP in diagnosis of HCC from CLD patients according to BCLC stage.

88.5 , and $200 \mathrm{ng} / \mathrm{mL}$ (90\% versus $40 \%, 20 \%$, and $0 \%$, resp., $P<0.001$ ) (Figure 2).

Both of MDK and AFP had excellent diagnostic performance to differentiate HCC group from the control group with no significant statistical difference regarding their AUC ( 0.998 versus $0.991 ; P=0.331)$, sensitivity (100\% versus $92.5 \%)$, and specificity (96.7\% versus $96.7 \%)$, respectively (Figure 3). The AUROC for combined serum MDK and AFP was 0.963 with $P<0.001$ and $95 \%$ CI (0.889 to 0.994$)$ (Figure 4).

\section{Discussion}

It has been estimated that $70 \%$ to $90 \%$ of patients with hepatocellular carcinomas have an established background of chronic liver disease or cirrhosis, the major causes of which are $\mathrm{HBV}$ or HCV infection [13]. In Egypt, a large study evaluated the epidemiological characteristics of HCC stated that HCV is the predominant cause of the underlying liver cirrhosis constituting about $91.32 \%$ of HCC cases while chronic HVB infection was reported in $2.51 \%$ [14]. This is very close to our results where $95 \%$ of HCC cases had chronic HCV and only $5 \%$ had chronic HBV infection.

When used as a diagnostic test, AFP levels at a value of $20 \mathrm{ng} / \mathrm{mL}$ show low specificity but fair sensitivity (60\%); that

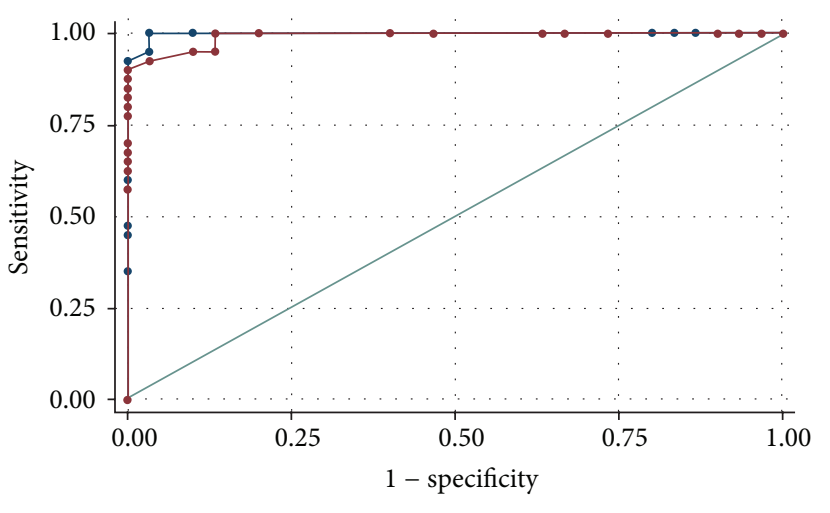

\begin{tabular}{lccccc}
\hline Test & AUC & $95 \%$ CI & $P$ & Sig. \\
\hline Midkine & 0.997 & 0.993 & 1.0 & & \\
$\begin{array}{l}\text { Alpha- } \\
\text { fetoprotein }\end{array}$ & 0.992 & 0.978 & 1.0 & & NS \\
\hline
\end{tabular}

- Midkine ROC area: 0.9979

Reference

- - Alpha-fetoprotein ROC area: 0.9912

FIGURE 3: ROC curve showing comparison of the diagnostic performance of serum MDK and AFP for discriminating HCC group from healthy group.

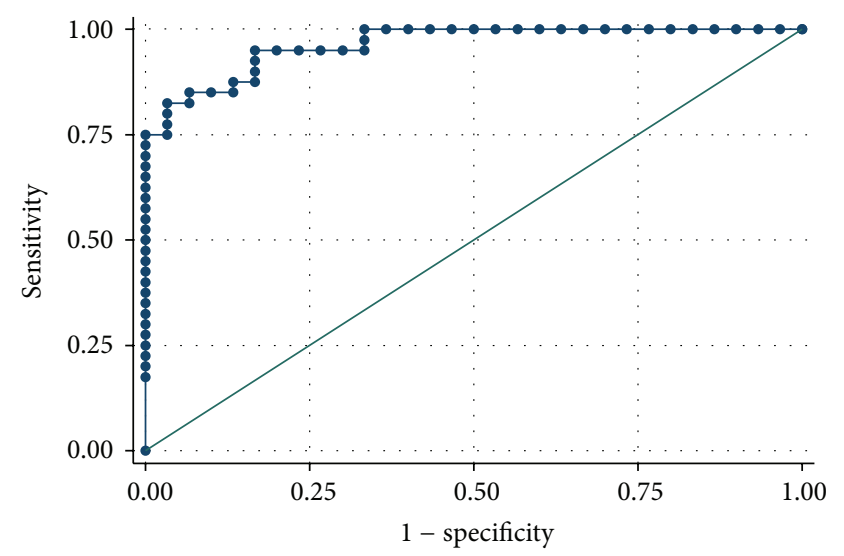

Area under ROC curve $=0.9633$

\begin{tabular}{lccc}
\hline Test & AUC & Standard error & 95\% confidence interval \\
\hline $\begin{array}{l}\text { Combined } \\
\text { midkine and } \\
\text { alpha-fetoprotein }\end{array}$ & 0.963 & 0.0221 & $0.889-0.994$ \\
\hline
\end{tabular}

FIGURE 4: ROC curve for combined midkine and alpha-fetoprotein for discrimination between HCC and CLD.

is, AFP surveillance would miss $40 \%$, whereas at higher cutoffs of $200 \mathrm{ng} / \mathrm{mL}$ the sensitivity drops to $22 \%$ with high specificity. Therefore, reducing the cutoff means that more HCCs would be identified, but at the cost of a progressive increase in the false-positive rate [15]. Consequently, AFP is an inadequate screening test [16] and is no longer assessed in surveillance programs due to the low capacity of identifying new cases not previously detected by imaging techniques [17]. These data are very similar from our findings as at cutoff 
values of $20 \mathrm{ng} / \mathrm{mL}$ and $200 \mathrm{ng} / \mathrm{mL}$ AFP had $62.5,25 \%$, sensitivity and $53.3,100 \%$, specificity, respectively.

In this study, we found serum MDK was significantly elevated in patients with hepatocellular carcinomas compared with liver cirrhosis patients (median: 0.625 versus $0.15 \mathrm{ng} / \mathrm{mL}$ ) and the healthy controls (median: 0.625 versus $0.125 \mathrm{ng} / \mathrm{mL}$ ). In addition, serum MDK was not significantly higher in the liver cirrhosis group than that in healthy group ( 0.15 versus $0.125 \mathrm{ng} / \mathrm{mL} ; P=1.559$ ), in contrast to serum AFP which was significantly elevated in the liver cirrhosis group when compared to healthy group (17.5 versus $1 \mathrm{ng} / \mathrm{mL}$; $P=0.0001)$. This means that the well-known nonspecific elevations of AFP in patients with liver cirrhosis were not significantly elicited with serum MDK increasing its specificity as a novel diagnostic marker for HCC.

Serum AFP levels were found to be significantly correlated with larger tumor size in this study $(r=0.36$; $P=$ $0.022)$. In addition, patients with advanced-stage hepatocellular carcinomas had significantly higher median AFP serum levels (BCLC B/C) than that of early-stage tumors (BCLC $0 / \mathrm{A})(300 \mathrm{ng} / \mathrm{mL}$ versus $17 \mathrm{ng} / \mathrm{mL}, P=0.0014)$. However, no significant correlation was found between serum MDK levels with tumor size, number or serum levels of AFP, and no significant association was found between serum MDK levels and BCLC stages.

The best cutoff values for MDK and AFP to discriminate HCC cases from those with liver cirrhosis were 0.387 and $88.5 \mathrm{ng} / \mathrm{mL}$, respectively, with sensitivities (92.5 versus $40 \%$ ), specificities ( 83.3 versus $96.7 \%$ ), and accuracies ( 88.5 versus $68.2 \%)$, respectively. Then through the analysis of the ROC, the AUC of MDK (0.941) was found to be much larger than that of serum AFP $(0.671 ; P<0.001)$ with high significant statistical difference. This means that the overall diagnostic performance of MDK for HCC diagnosis is much better than that of AFP.

On comparing the sensitivities and specificities of MDK at the cutoff $0.387 \mathrm{ng} / \mathrm{mL}$ to those of AFP at different cutoff values $(20,88.5$, and $200 \mathrm{ng} / \mathrm{mL})$, we found that the sensitivities of MDK were significantly higher than those of AFP at all values ( 92.5 versus $62.5,40$, and $25 \%$ ), respectively, with similar specificities to AFP at cutoffs 88.5 and $200 \mathrm{ng} / \mathrm{mL}$, while MDK had significantly higher specificity than that of AFP only at the value of $20 \mathrm{ng} / \mathrm{mL}$ (83.3 versus 53.3).

Fifteen out of 40 HCC patients (37\%) had low AFP $(<20 \mathrm{ng})$; from those 15 patients, only 1 patient had MDK $<0.387 \mathrm{ng} / \mathrm{mL}$; this shows that MDK had an outstanding performance for distinguishing hepatocellular carcinomas from liver cirrhosis even in patients with AFP $<20$ with sensitivity reaching $93.3 \%$. No one in the healthy control group exceeded MDK cutoff level $0.38 \mathrm{ng} / \mathrm{mL}$ and only 5 patients (16.7\%) from liver cirrhosis group exceeded this threshold. On the other hand, $46.7 \%$ (14 of 30) of these patients were above the cutoff value of AFP $(20 \mathrm{ng} / \mathrm{mL})$. These indicate that MDK is a novel marker and superior to AFP with a lower falsepositive rate in diagnosing and differentiating hepatocellular carcinomas from liver cirrhosis.

In terms of early detection and diagnosis of HCC, MDK at cutoff value $0.387 \mathrm{ng} / \mathrm{mL}$ showed a superior diagnostic performance to differentiate early stage HCC from patients with liver cirrhosis when compared to AFP at different cutoff values $20,88.5$, and $200 \mathrm{ng} / \mathrm{mL}$ (BCLC 0/A; sensitivity, $90 \%$ versus 40,20 , and $0 \%$, resp. $)(P<0.001)$.

The AUC of combined MDK and AFP for discrimination between HCC and liver cirrhosis patients was larger than that MDK alone (0.963 versus 0.941$)$ but the difference did not reach a significant level. Thus, combination of MDK and APF may be a promising strategy for early diagnosis of hepatocellular carcinomas in the future.

Our results are close to those reported by Zhu et al. [18]. That study involved three independent cohorts with a total of 933 participants including 388 HCC cases and 545 different controls enrolled from different medical centers. Results showed that MDK levels were significantly elevated in HCC tissues as well as serum samples; serum MDK at the cutoff value of $0.654 \mathrm{ng} / \mathrm{mL}$ for HCC diagnosis showed an obviously higher sensitivity compared with AFP (86.9\% versus $51.9 \%$ ) with similar specificities ( $83.9 \%$ versus $86.3 \%$ ); even in very early-stage HCC, the sensitivity of MDK was significant higher than AFP ( $80 \%$ versus 40\%); in those AFP-negative HCC cases, the sensitivity could reach as high as $89.2 \%$; and serum MDK level was significantly decreased in HCC patients after curative resection and reelevated when tumor relapsed [18]. But the value of MDK in detection of HCVrelated early HCC was not analyzed in that study, which was investigated in the current study.

In conclusion, serum MDK may serve as a novel diagnostic tumor marker for the detection of hepatocellular carcinomas, particularly in patients with AFP $<20 \mathrm{ng} / \mathrm{mL}$ and/or at an early stage. Further studies with larger population are needed to justify its implementation in clinical practice.

\section{Conflict of Interests}

The authors declare that there is no conflict of interests regarding the publication of this paper.

\section{References}

[1] D. M. Parkin, F. Bray, J. Ferlay, and P. Pisani, "Global cancer statistics, 2002," CA: A Cancer Journal for Clinicians, vol. 55, no. 2, pp. 74-108, 2005.

[2] NCI hospital based registry 2002-2010 by Nelly Alieldin, The National Cancer Institute, Cairo, Egypt, http://www.nci.cu.edu .eg/.

[3] European Association for the Study of the Liver and European Organisation for Research and Treatment of Cancer, "EASLEORTC clinical practice guidlines: managment of hepatocellular carcinoma," Journal of Hepatology, vol. 56, no. 4, pp. 908943, 2012.

[4] A. M. di Bisceglie, R. K. Sterling, R. T. Chung et al., "Serum alpha-fetoprotein levels in patients with advanced hepatitis $\mathrm{C}$ : results from the HALT-C Trial," Journal of Hepatology, vol. 43, no. 3, pp. 434-441, 2005.

[5] T. Yamashita, M. Forgues, W. Wang et al., "EpCAM and alphafetoprotein expression defines novel prognostic subtypes of hepatocellular carcinoma," Cancer Research, vol. 68, no. 5, pp. 1451-1461, 2008.

[6] A. Villanueva, B. Minguez, A. Forner, M. Reig, and J. M. Llovet, "Hepatocellular carcinoma: novel molecular approaches for 
diagnosis, prognosis, and therapy," Annual Review of Medicine, vol. 61, pp. 317-328, 2010.

[7] Y. Koike, Y. Shiratori, S. Sato et al., "Des- $\gamma$-carboxy prothrombin as a useful predisposing factor for the development of portal venous invasion in patients with hepatocellular carcinoma: a prospective analysis of 227 patients," Cancer, vol. 91, no. 3, pp. 561-569, 2001.

[8] R. K. Sterling, L. Jeffers, F. Gordon et al., "Clinical utility of AFP-L3\% measurement in North American patients with HCVrelated cirrhosis," The American Journal of Gastroenterology, vol. 102, no. 10, pp. 2196-2205, 2007.

[9] M. Ibusuki, H. Fujimori, Y. Yamamoto et al., "Midkine in plasma as a novel breast cancer marker," Cancer Science, vol. 100, no. 9, pp. 1735-1739, 2009.

[10] M. Kato, T. Shinozawa, S. Kato, A. Awaya, and T. Terada, "Increased midkine expression in hepatocellular carcinoma," Archives of Pathology and Laboratory Medicine, vol. 124, no. 6, pp. 848-852, 2000.

[11] T. Muramatsu, "Midkine and pleiotrophin: two related proteins involved in development, survival, inflammation and tumorigenesis," Journal of Biochemistry (Tokyo), vol. 132, no. 3, pp. 359371, 2002.

[12] J. Bruix and M. Sherman, "Management of hepatocellular carcinoma: an update," Hepatology, vol. 53, no. 3, pp. 1020-1022, 2011.

[13] H. B. El-Serag and K. L. Rudolph, "Hepatocellular carcinoma: epidemiology and molecular carcinogenesis," Gastroenterology, vol. 132, no. 7, pp. 2557-2576, 2007.

[14] M. K. Shaker, H. M. Abdella, M. O. Khalifa, and A. K. ElDorry, "Epidemiological characteristics of hepatocellular carcinoma in Egypt: a retrospective analysis of 1313 cases," Liver International, vol. 33, no. 10, pp. 1601-1606, 2013.

[15] F. Trevisani, P. E. D’Intino, A. M. Morselli-Labate et al., "Serum alpha-fetoprotein for diagnosis of hepatocellular carcinoma in patients with chronic liver disease: influence of HBsAg and antiHCV status," Journal of Hepatology, vol. 34, no. 4, pp. 570-575, 2001.

[16] M. Sherman, "Alphafetoprotein: an obituary," Journal of Hepatology, vol. 34, no. 4, pp. 603-605, 2001.

[17] J. M. Llovet and J. Bruix, "Novel advancements in the management of hepatocellular carcinoma in 2008," Journal of Hepatology, vol. 48, pp. S20-S37, 2008.

[18] W.-W. Zhu, J.-J. Guo, L. Guo et al., "Evaluation of midkine as a diagnostic serum biomarker in hepatocellular carcinoma," Clinical Cancer Research, vol. 19, no. 14, pp. 3944-3954, 2013. 


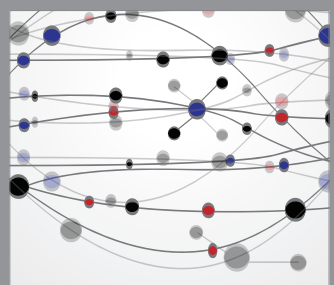

The Scientific World Journal
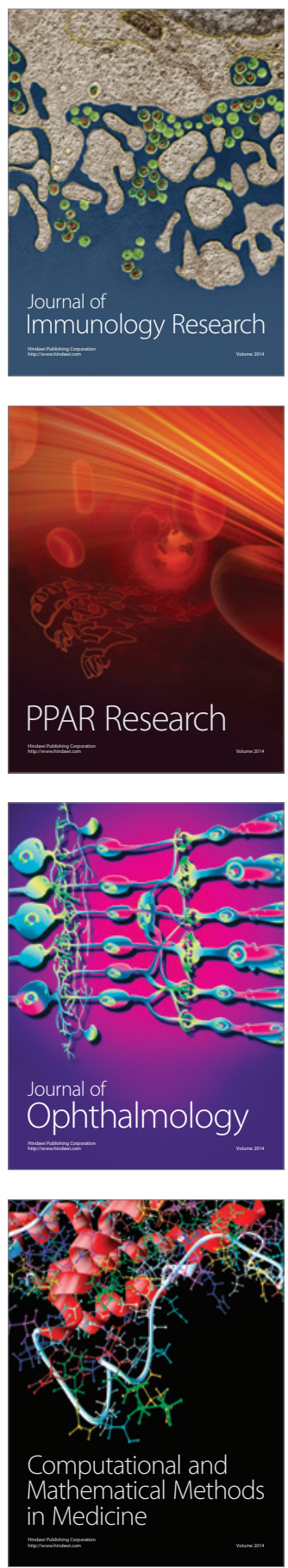

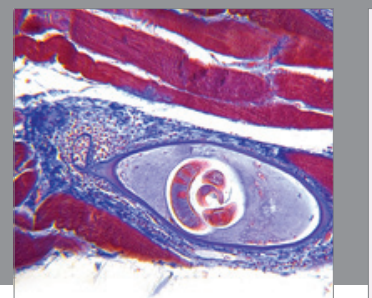

Gastroenterology

Research and Practice
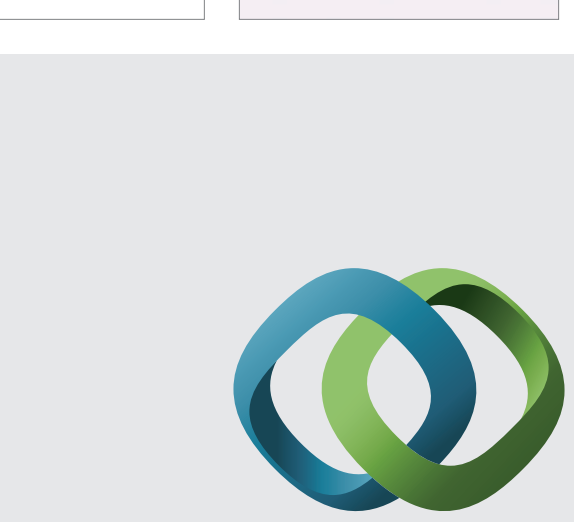

\section{Hindawi}

Submit your manuscripts at

http://www.hindawi.com
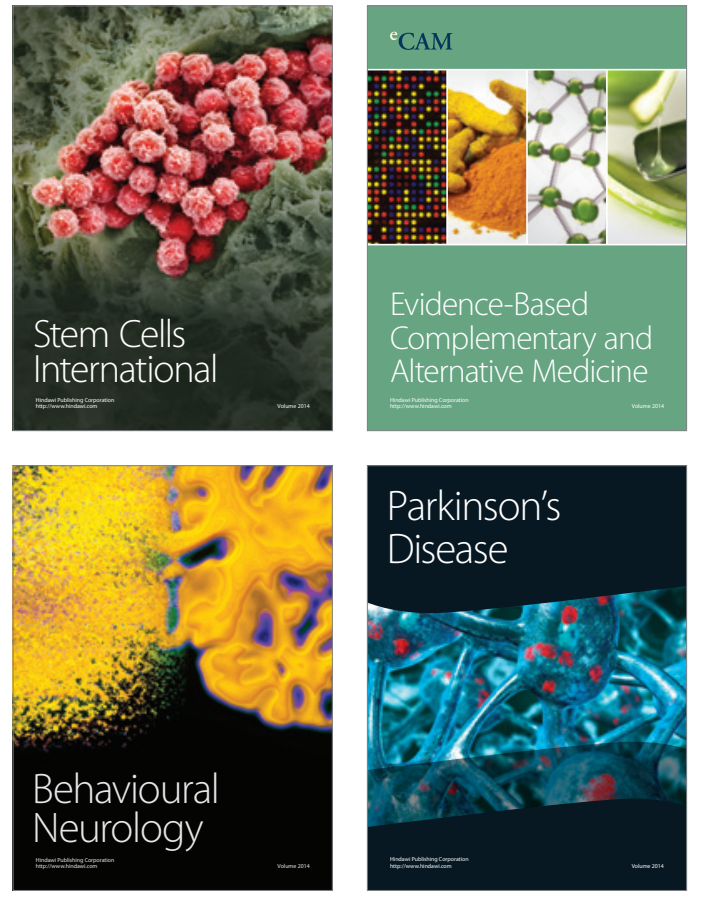
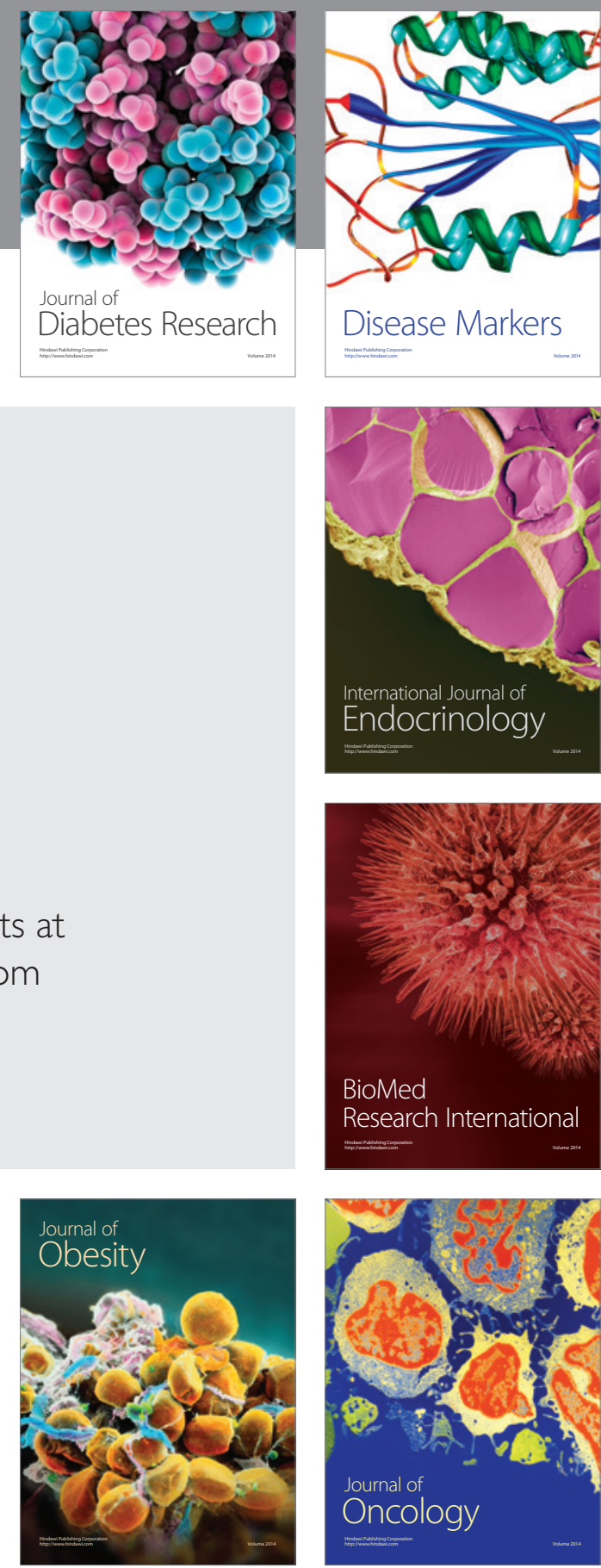

Disease Markers
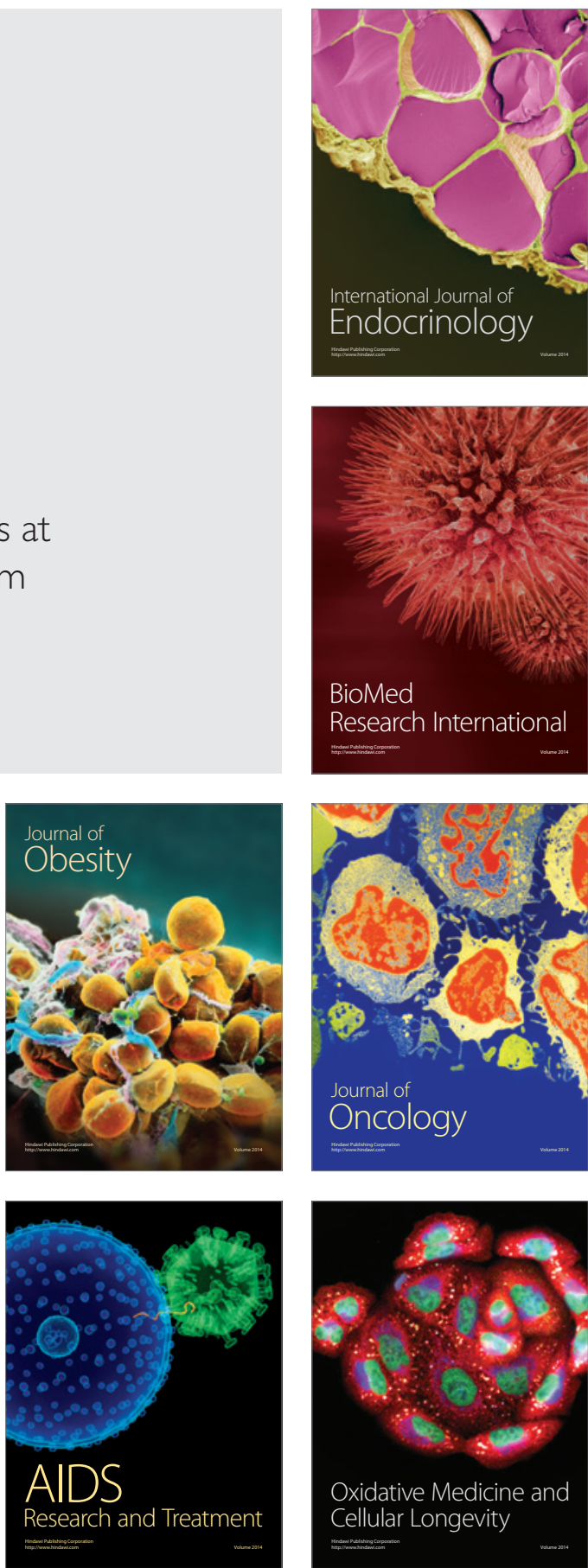